\title{
Histotype-Dependent Oligodendroglial PrP Pathology in Sporadic CJD: A Frequent Feature of the M2C "Strain"
}

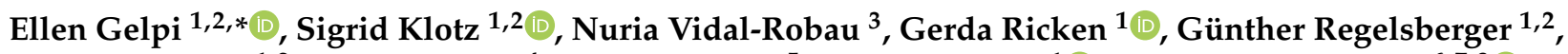

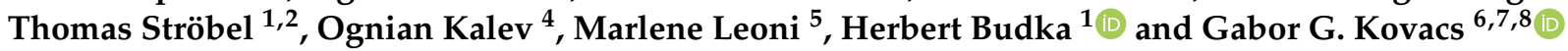

1 Division of Neuropathology and Neurochemistry, Department of Neurology, Medical University of Vienna, 1090 Vienna, Austria; sigrid.klotz@meduniwien.ac.at (S.K.); gerda.ricken@meduniwien.ac.at (G.R.); guenther.regelsberger@meduniwien.ac.at (G.R.); thomas.stroebel@meduniwien.ac.at (T.S.); herbert.budka@meduniwien.ac.at (H.B.)

2 Medical University of Vienna, Austrian Reference Center for Human Prion Diseases (OERPE), 1090 Vienna, Austria

3 Department of Neurology, Bellvitge University Hospital, L'Hospitalet de Llobregat, 08908 Barcelona, Spain; nuria.virob@gmail.com

4 Department of Neuropathology/Pathology, Kepler Universitäts Klinikum, 4020 Linz, Austria; Ognian.Kalev@kepleruniklinikum.at

5 Department of Neuropathology/Pathology, Medical University Graz, 8036 Graz, Austria; marlene.leoni@medunigraz.at

6 Tanz Centre for Research in Neurodegenerative Disease, University of Toronto, Toronto, ON M5T 0S8, Canada; Gabor.Kovacs@uhnresearch.ca

check for updates

Citation: Gelpi, E.; Klotz, S.; Vidal-Robau, N.; Ricken, G.; Regelsberger, G.; Ströbel, T.; Kalev, O.; Leoni, M.; Budka, H.; Kovacs, G.G. Histotype-Dependent Oligodendroglial PrP Pathology in Sporadic CJD: A Frequent Feature of the M2C "Strain". Viruses 2021, 13, 1796. https://doi.org/10.3390/ v13091796

Academic Editor: Valerie L. Sim

Received: 11 July 2021

Accepted: 2 September 2021

Published: 9 September 2021

Publisher's Note: MDPI stays neutral with regard to jurisdictional claims in published maps and institutional affiliations.

Copyright: (c) 2021 by the authors. Licensee MDPI, Basel, Switzerland. This article is an open access article distributed under the terms and conditions of the Creative Commons Attribution (CC BY) license (https:/ / creativecommons.org/licenses/by/ $4.0 /)$.
7 Department of Laboratory Medicine and Pathobiology and Department of Medicine, University of Toronto, Toronto, ON M5T 0S8, Canada

8 Laboratory Medicine Program \& Krembil Brain Institute, University Health Network, Toronto, ON M5T 1M8, Canada

* Correspondence: ellen.gelpi@meduniwien.ac.at; Tel.: +43-1-404063330

\begin{abstract}
In sporadic Creutzfeldt-Jakob disease, molecular subtypes are neuropathologically well identified by the lesioning profile and the immunohistochemical $\mathrm{PrP}^{\mathrm{d}}$ deposition pattern in the grey matter (histotypes). While astrocytic PrP pathology has been reported in variant CJD and some less frequent histotypes (e.g., MV2K), oligodendroglial pathology has been rarely addressed. We assessed a series of SCJD cases with the aim to identify particular histotypes that could be more prone to harbor oligodendroglial $\mathrm{PrP}^{\mathrm{d}}$. Particularly, the MM2C phenotype, in both its more "pure" and its mixed $\mathrm{MM} 1+2 \mathrm{C}$ or $\mathrm{MV} 2 \mathrm{~K}+2 \mathrm{C}$ forms, showed more frequent oligodendroglial PrP pathology in the underlying white matter than the more common MM1/MV1 and VV2 histotypes, and was more abundant in patients with a longer disease duration. We concluded that the MM2C strain was particularly prone to accumulate $\mathrm{PrP}^{\mathrm{d}}$ in white matter oligodendrocytes.
\end{abstract}

Keywords: Creutzfeldt-Jakob disease; CJD; PrP; prion; histotype; glia; oligodendrocytes

\section{Introduction}

In human prion diseases, attention has been mainly focused on neuronal dysfunction, as $\operatorname{PrP}^{\mathrm{C}}$ is enriched in synapses and its pathology positively correlates with the neurodegenerative process and clinical symptoms [1]. Astroglial pathology is an important feature of variant CJD and pathological astroglial PrP deposits represent, in addition to florid plaques, a key histopathological hallmark [2]. Moreover, the deposition of disease-associated PrP $\left(\mathrm{PrP}^{\mathrm{d}}\right)$ has been described in astrocytes [3], particularly in MV2K cases [4]. In contrast, oligodendrocytic PrP pathology has rarely been described. Fernandez-Vega et al. [5] reported the presence of nuclear and perinuclear $\mathrm{PrP}^{\mathrm{d}}$ in oligodendrocytes in the frontal white matter in a 66-year-old man with an otherwise classical VV2-histotype, who had a disease duration of 4,5 months. We also observed the presence of oligodendroglial $\mathrm{PrP}^{\mathrm{d}}$ pathology in the white matter of some sporadic CJD cases. This finding prompted us 
to analyze in more detail whether some specific CJD histotypes might be more prone to accumulate $\operatorname{PrP}^{\mathrm{d}}$ in this particular glial cell type.

\section{Materials and Methods}

We screened the different basic histotypes of sporadic CJD: $20 \mathrm{MM} / \mathrm{MV} 1,10 \mathrm{VV} 2$, 15 mixed MM/MV1 + 2C, 10 mixed MV2K + 2C and 10 MM2C. Demographical details of the patients and disease duration are given in Table 1. We assessed formalin-fixed, formic-acid-decontaminated, and paraffin-embedded tissue sections from the frontal and occipital lobe and paid particular attention to white matter pathology. Immunohistochemistry was performed by applying the anti-PrP 12F10 antibody (1:1000, epitope aa 142-160, CEA, Gif-sur-Yvette Cedex, France), after appropriate tissue pretreatment. This included a three-tiered tissue pretreatment based on $10 \mathrm{~min}$ hydrated autoclaving at $121^{\circ} \mathrm{C}, 5 \mathrm{~min}$, $96 \%$ formic acid, and $5 \mathrm{~min}$ proteinase $\mathrm{K}\left(5 \mu \mathrm{g} / \mathrm{mL}\right.$ in TRIS) at $4{ }^{\circ} \mathrm{C}$, prior to anti-PrP antibody incubation. In parallel we evaluated the $\mathrm{PrP}^{\mathrm{d}}$ deposition pattern in the overlying cortex as synaptic, perineuronal, patchy perivacuolar, plaque-like and/or Kuru-type plaques, and assessed its intensity in a semiquantitative scale as follows: $0=$ absent, $1=$ mild, 2 = moderate, 3 = extensive. In selected cases that harbored oligodendroglial $\mathrm{PrP}^{\mathrm{d}}$ pathology, we extended the anti-PrP antibody panel and included the monoclonal antibody 3F4 (1:500, epitope 109-112, Senetek PLC, CA, USA), 6H4 (1:500, epitope 144-152, Prionics, Schlieren ZH, Switzerland), KG9 (1:1000, epitope 140-180, TSE Resource Centre, Edinburgh, UK), and L42 (1:300, epitope 141-159 IgG1 FRC for Virus Diseases of Animals, sheep recPrP; Dr. M.H. Groschup, Tübingen, Germany). The Dako Envision Kit (DAKO, Glostrup, Denmark) was used as secondary system and diaminobenzidine as chromogen. Double immunofluorescence was performed on selected cases combining anti-PrP 12F10 and the oligodendroglia marker anti-TPPP/p25 (anti-tubulin polymerization promoting protein TPPP/p25, a protein that was expressed mainly in differentiated oligodendrocytes of the CNS [6], non-commercial antibody, rabbit, 1:250) as well as anti-PrP $12 \mathrm{~F} 10$ and anti-GFAP antibodies (rabbit, 1:1500, DAKO), applying Alexa Fluor 488 goat-anti-mouse antibody (1:800, Jackson Immunoresearch, PA, USA) and Cy3 goat-anti-rabbit (1:1000, Jackson Immunoresearch, PA, USA) as secondary antibodies, in addition to DAPI nuclear stain $(1 \mu \mathrm{g} / \mathrm{mL}$, Thermo Fisher Scientific, MA, USA). Incubation of the antibodies was performed overnight at $4{ }^{\circ} \mathrm{C}$. Autofluorescence was blocked with $1 \%$ aqueous sodium borohydride solution ( $4 \mathrm{~min}$ ) and $1 \%$ sudan black B solution ( $5 \mathrm{~min}$ ).

Table 1. Demographic and neuropathological characteristics of patients included in the study.

\begin{tabular}{|c|c|c|c|c|c|c|c|c|}
\hline Case no. & Sex & $\begin{array}{l}\text { Age at } \\
\text { Death } \\
\text { (years) }\end{array}$ & $\begin{array}{l}\text { Disease } \\
\text { Duration } \\
\text { (months) }\end{array}$ & Histotype & $\begin{array}{c}\text { Oligodendroglial } \\
\text { PrPd White }^{d} \text { Watter }\end{array}$ & $\begin{array}{c}\text { Main PrP } \\
\text { Pattern Frontal }\end{array}$ & $\begin{array}{c}\text { Main PrPd } \\
\text { Pattern Occipital }\end{array}$ & $\begin{array}{l}\text { Intensity PrPd } \\
\text { Deposits }\end{array}$ \\
\hline 1 & $\mathrm{f}$ & 72 & 3 & MM/MV1 & no & diffuse synaptic & diffuse synaptic & extensive \\
\hline 2 & $\mathrm{~m}$ & 71 & 3 & MM/MV1 & no & $\begin{array}{l}\text { diffuse synaptic } \\
\text { deep laminar }\end{array}$ & diffuse synaptic & $\begin{array}{c}\text { mild frontal, } \\
\text { extensive } \\
\text { occipital } \\
\end{array}$ \\
\hline 3 & $\mathrm{~m}$ & 72 & 3 & MM/MV1 & $\begin{array}{l}\text { isolated frontal } \\
\text { (severe cortical } \\
\text { degeneration) }\end{array}$ & diffuse synaptic & diffuse synaptic & extensive \\
\hline 4 & $\mathrm{~m}$ & 73 & 5 & MM/MV1 & O & $\begin{array}{l}\text { Diffuse synaptic } \\
\text { deep laminar }\end{array}$ & $\begin{array}{l}\text { diffuse synaptic } \\
\text { deep laminar }\end{array}$ & moderate \\
\hline 5 & $\mathrm{f}$ & 63 & 1.5 & MM/MV1 & no & diffuse synaptic & diffuse synaptic & extensive \\
\hline 6 & $\mathrm{~m}$ & 62 & 6 & MM/MV1 & no & $\begin{array}{l}\text { diffuse synaptic } \\
\text { laminar }\end{array}$ & $\begin{array}{l}\text { diffuse synaptic } \\
\text { laminar }\end{array}$ & mild \\
\hline 7 & $\mathrm{f}$ & 57 & 3 & MM/MV1 & no & diffuse synaptic & diffuse synaptic & extensive \\
\hline 8 & $\mathrm{~m}$ & 75 & 2 & MM/MV1 & no & diffuse synaptic & diffuse synaptic & mild \\
\hline 9 & $\mathrm{~m}$ & 72 & 2 & MM/MV1 & no & diffuse synaptic & diffuse synaptic & $\begin{array}{l}\text { moderate } \\
\text { frontal, } \\
\text { extensive } \\
\text { occipital }\end{array}$ \\
\hline 10 & $\mathrm{~m}$ & 72 & 2 & MM/MV1 & no & diffuse synaptic & diffuse synaptic & moderate \\
\hline 11 & $\mathrm{f}$ & 74 & 6 & MM/MV1 & no & diffuse synaptic & diffuse synaptic & extensive \\
\hline 12 & $\mathrm{f}$ & 67 & 2 & MM/MV1 & no & diffuse synaptic & diffuse synaptic & moderate \\
\hline 13 & $\mathrm{~m}$ & 63 & 1 & MM/MV1 & no & diffuse synaptic & diffuse synaptic & moderate \\
\hline 14 & $\mathrm{f}$ & 67 & 3 & MM/MV1 & no & diffuse synaptic & diffuse synaptic & moderate \\
\hline
\end{tabular}


Table 1. Cont.

\begin{tabular}{|c|c|c|c|c|c|c|c|c|}
\hline Case no. & Sex & $\begin{array}{l}\text { Age at } \\
\text { Death } \\
\text { (years) }\end{array}$ & $\begin{array}{l}\text { Disease } \\
\text { Duration } \\
\text { (months) }\end{array}$ & Histotype & $\begin{array}{c}\text { Oligodendroglial } \\
\text { PrP }^{\mathrm{d}} \text { White } \\
\text { Matter } \\
\end{array}$ & $\begin{array}{c}\text { Main PrP } \\
\text { Pattern Frontal }\end{array}$ & $\begin{array}{c}\text { Main PrPd } \\
\text { Pattern Occipital }\end{array}$ & $\begin{array}{c}\text { Intensity } \operatorname{PrP}^{\mathrm{d}} \\
\text { Deposits }\end{array}$ \\
\hline 15 & $\mathrm{~m}$ & 59 & 3.5 & MM/MV1 & no & diffuse synaptic & diffuse synaptic & $\begin{array}{c}\text { moderate } \\
\text { frontal, } \\
\text { extensive } \\
\text { occipital }\end{array}$ \\
\hline 16 & $\mathrm{~m}$ & 56 & 2 & MM/MV1 & no & diffuse synaptic & diffuse synaptic & moderate \\
\hline 17 & $\mathrm{f}$ & 65 & 2 & MM/MV1 & no & diffuse synaptic & diffuse synaptic & extensive \\
\hline 18 & $\mathrm{f}$ & 54 & 2 & MM/MV1 & no & diffuse synaptic & diffuse synaptic & $\begin{array}{l}\text { moderate, } \\
\text { extensive } \\
\text { occipital }\end{array}$ \\
\hline 19 & $\mathrm{~m}$ & 77 & 2 & MM/MV1 & no & diffuse synaptic & diffuse synaptic & $\begin{array}{l}\text { extensive } \\
\text { frontal, mild } \\
\text { occipital }\end{array}$ \\
\hline 20 & $\mathrm{f}$ & 65 & 1.5 & MM/MV1 & no & diffuse synaptic & diffuse synaptic & moderate \\
\hline 1 & $\mathrm{f}$ & 66 & 5 & VV2 & no & $\begin{array}{c}\text { deep } \\
\text { perineuronal }\end{array}$ & deep perineuronal & moderate \\
\hline 2 & $\mathrm{~m}$ & 62 & 3 & VV2 & no & $\begin{array}{c}\text { deep } \\
\text { perineuronal }\end{array}$ & deep perineuronal & moderate \\
\hline 3 & $\mathrm{~m}$ & 62 & 4 & VV2 & no & $\begin{array}{c}\text { deep } \\
\text { perineuronal }\end{array}$ & deep perineuronal & extensive \\
\hline 4 & $\mathrm{f}$ & 69 & 6 & VV2 & no & $\begin{array}{c}\text { deep } \\
\text { perineuronal }\end{array}$ & $\begin{array}{l}\text { deep perineuronal } \\
+ \text { plaque-like }\end{array}$ & extensive \\
\hline 5 & $\mathrm{f}$ & 74 & 3 & VV2 & no & $\begin{array}{c}\text { deep } \\
\text { perineuronal + } \\
\text { plaque-like }\end{array}$ & $\begin{array}{l}\text { deep perineuronal } \\
+ \text { plaque-like }\end{array}$ & moderate \\
\hline 6 & $\mathrm{~m}$ & 81 & 2 & VV2 & no & $\begin{array}{c}\text { deep } \\
\text { perineuronal + } \\
\text { plaque-like }\end{array}$ & deep perineuronal & mild \\
\hline 7 & $\mathrm{~m}$ & 74 & 3 & VV2 & no & $\begin{array}{c}\text { deep } \\
\text { perineuronal + } \\
\text { plaque-like }\end{array}$ & $\begin{array}{l}\text { deep perineuronal } \\
\quad+\text { plaque-like }\end{array}$ & $\begin{array}{c}\text { extensive } \\
\text { frontal, mild } \\
\text { occipital }\end{array}$ \\
\hline 8 & $\mathrm{~m}$ & 78 & 4 & VV2 & no & $\begin{array}{c}\text { deep } \\
\text { perineuronal }\end{array}$ & deep perineuronal & $\begin{array}{c}\text { extensive } \\
\text { frontal, } \\
\text { moderate } \\
\text { occipital }\end{array}$ \\
\hline 9 & $\mathrm{f}$ & 75 & 5 & VV2 & no & $\begin{array}{c}\text { deep } \\
\text { perineuronal }\end{array}$ & deep perineuronal & $\begin{array}{l}\text { moderate } \\
\text { frontal, mild } \\
\text { occipital }\end{array}$ \\
\hline 10 & $\mathrm{f}$ & 80 & 3 & VV2 & no & $\begin{array}{c}\text { deep } \\
\text { perineuronal }\end{array}$ & deep perineuronal & moderate \\
\hline 1 & $\mathrm{~m}$ & 74 & 7 & $\mathrm{MM} / \mathrm{MV} 1+2 \mathrm{C}$ & yes, isol & $\begin{array}{l}\text { diffuse synaptic } \\
+ \text { focal patchy }\end{array}$ & $\begin{array}{l}\text { diffuse synaptic + } \\
\text { patchy }\end{array}$ & $\begin{array}{l}\text { moderate- } \\
\text { extensive }\end{array}$ \\
\hline 2 & $\mathrm{~m}$ & 65 & 17 & $\mathrm{MM} / \mathrm{MV} 1+2 \mathrm{C}$ & no & diffuse synaptic & $\begin{array}{l}\text { diffuse synaptic }+ \\
\text { focal patchy }\end{array}$ & $\begin{array}{c}\text { extensive } \\
\text { synaptic } \\
\text { frontal, mild } \\
\text { synaptic } \\
\text { occipital } \\
\end{array}$ \\
\hline 3 & $\mathrm{f}$ & 77 & 2 & $\mathrm{MM} / \mathrm{MV} 1+2 \mathrm{C}$ & no & diffuse synaptic & $\begin{array}{l}\text { diffuse synaptic + } \\
\text { focal patchy }\end{array}$ & moderate \\
\hline 4 & $\mathrm{~m}$ & 59 & 3 & $\mathrm{MM} / \mathrm{MV} 1+2 \mathrm{C}$ & no & $\begin{array}{l}\text { diffuse synaptic } \\
+ \text { focal patchy }\end{array}$ & $\begin{array}{l}\text { diffuse synaptic }+ \\
\text { focal patchy }\end{array}$ & $\begin{array}{l}\text { mild synaptic } \\
\text { frontal, } \\
\text { moderate } \\
\text { synaptic } \\
\text { occipital }\end{array}$ \\
\hline 5 & $\mathrm{~m}$ & 81 & 2 & $\mathrm{MM} / \mathrm{MV} 1+2 \mathrm{C}$ & no & diffuse synaptic & $\begin{array}{l}\text { diffuse synaptic }+ \\
\text { focal patchy }\end{array}$ & mild synaptic \\
\hline 6 & $\mathrm{~m}$ & 55 & 9 & $\mathrm{MM} / \mathrm{MV} 1+2 \mathrm{C}$ & no & $\begin{array}{l}\text { diffuse synaptic } \\
+ \text { patchy }\end{array}$ & diffuse synaptic & $\begin{array}{c}\text { moderate } \\
\text { frontal, } \\
\text { extensive } \\
\text { synaptic } \\
\text { occipital }\end{array}$ \\
\hline 7 & $\mathrm{f}$ & 68 & 1 & $\mathrm{MM} / \mathrm{MV} 1+2 \mathrm{C}$ & no & $\begin{array}{l}\text { diffuse synaptic } \\
+ \text { focal patchy }\end{array}$ & $\begin{array}{c}\text { diffuse synaptic + } \\
\text { focal patchy }\end{array}$ & $\begin{array}{c}\text { extensive } \\
\text { synaptic }\end{array}$ \\
\hline 8 & $\mathrm{f}$ & 51 & 5 & $\mathrm{MM} / \mathrm{MV} 1+2 \mathrm{C}$ & yes, few frontal & $\begin{array}{c}\text { patchy } \\
\text { perivacuolar }\end{array}$ & $\begin{array}{l}\text { diffuse synaptic }+ \\
\text { focal patchy }\end{array}$ & $\begin{array}{c}\text { extensive } \\
\text { patchy frontal, } \\
\text { extensive } \\
\text { synaptic } \\
\text { occipital }\end{array}$ \\
\hline 9 & $\mathrm{f}$ & 79 & 5 & $\mathrm{MM} / \mathrm{MV} 1+2 \mathrm{C}$ & yes, few frontal & $\begin{array}{c}\text { patchy } \\
\text { perivacuolar }\end{array}$ & $\begin{array}{l}\text { diffuse synaptic }+ \\
\text { focal patchy }\end{array}$ & $\begin{array}{c}\text { extensive } \\
\text { patchy frontal, } \\
\text { extensive } \\
\text { synaptic } \\
\text { occipital }\end{array}$ \\
\hline
\end{tabular}


Table 1. Cont

\begin{tabular}{|c|c|c|c|c|c|c|c|c|}
\hline Case no. & Sex & $\begin{array}{l}\text { Age at } \\
\text { Death } \\
\text { (years) }\end{array}$ & $\begin{array}{l}\text { Disease } \\
\text { Duration } \\
\text { (months) }\end{array}$ & Histotype & $\begin{array}{l}\text { Oligodendroglial } \\
\text { PrPd White }^{\text {d }} \\
\text { Matter } \\
\end{array}$ & $\begin{array}{c}\text { Main PrP } \\
\text { Pattern Frontal }\end{array}$ & $\begin{array}{c}\text { Main PrP } \\
\text { Pattern Occipital }\end{array}$ & $\begin{array}{c}\text { Intensity } \operatorname{PrP}^{\mathrm{d}} \\
\text { Deposits }\end{array}$ \\
\hline 10 & $\mathrm{~m}$ & 74 & 2 & $\mathrm{MM} / \mathrm{MV} 1+2 \mathrm{C}$ & no & $\begin{array}{l}\text { diffuse synaptic } \\
+ \text { focal patchy }\end{array}$ & $\begin{array}{c}\text { diffuse synaptic }+ \\
\text { focal patchy }\end{array}$ & $\begin{array}{l}\text { moderate } \\
\text { synaptic }\end{array}$ \\
\hline 11 & $\mathrm{~m}$ & 70 & 2 & $\mathrm{MM} / \mathrm{MV} 1+2 \mathrm{C}$ & no & diffuse synaptic & $\begin{array}{l}\text { diffuse synaptic }+ \\
\text { focal patchy }\end{array}$ & $\begin{array}{c}\text { extensive } \\
\text { synaptic } \\
\text { frontal, } \\
\text { moderate } \\
\text { synaptic } \\
\text { occipital }\end{array}$ \\
\hline 12 & $\mathrm{~m}$ & 62 & 5 & $\mathrm{MM} / \mathrm{MV} 1+2 \mathrm{C}$ & no & diffuse synaptic & $\begin{array}{l}\text { diffuse synaptic }+ \\
\text { focal patchy }\end{array}$ & extensive \\
\hline 13 & $\mathrm{~m}$ & 62 & 2 & MM/MV1+2C & no & diffuse synaptic & diffuse synaptic & moderate \\
\hline 14 & $\mathrm{f}$ & 55 & 2 & $\mathrm{MM} / \mathrm{MV} 1+2 \mathrm{C}$ & yes, few occipital & $\begin{array}{l}\text { diffuse synaptic } \\
+ \text { patchy }\end{array}$ & $\begin{array}{l}\text { diffuse synaptic }+ \\
\text { patchy }\end{array}$ & $\begin{array}{c}\text { moderate } \\
\text { frontal, } \\
\text { extensive } \\
\text { patchy } \\
\text { occipital }\end{array}$ \\
\hline 15 & $\mathrm{f}$ & 96 & n.a. & $\mathrm{MM} / \mathrm{MV} 1+2 \mathrm{C}$ & no & $\begin{array}{l}\text { diffuse synaptic } \\
+ \text { focal patchy }\end{array}$ & $\begin{array}{l}\text { diffuse synaptic }+ \\
\text { patchy }\end{array}$ & $\begin{array}{l}\text { moderate- } \\
\text { extensive }\end{array}$ \\
\hline 1 & $\mathrm{~m}$ & 66 & 3 & $\mathrm{MV} 2 \mathrm{~K}+\mathrm{C}$ & no & mild & mild & mild \\
\hline 2 & $\mathrm{~m}$ & 62 & 7 & $\mathrm{MV} 2 \mathrm{~K}+\mathrm{C}$ & yes & $\begin{array}{c}\text { patchy } \\
\text { perivacuolar }\end{array}$ & $\begin{array}{c}\text { patchy } \\
\text { perivacuolar }\end{array}$ & extensive \\
\hline 3 & $\mathrm{f}$ & 62 & 13 & $\mathrm{MV} 2 \mathrm{~K}+\mathrm{C}$ & no & $\begin{array}{c}\text { patchy } \\
\text { perivacuolar }\end{array}$ & $\begin{array}{c}\text { patchy } \\
\text { perivacuolar }\end{array}$ & moderate \\
\hline 4 & $\mathrm{~m}$ & 78 & 8 & $\mathrm{MV} 2 \mathrm{~K}+\mathrm{C}$ & yes, few occipital & $\begin{array}{c}\text { patchy } \\
\text { perivacuolar }\end{array}$ & $\begin{array}{c}\text { patchy } \\
\text { perivacuolar }\end{array}$ & extensive \\
\hline 5 & $\mathrm{f}$ & 77 & 12 & $\mathrm{MV} 2 \mathrm{~K}+\mathrm{C}$ & yes, few occipital & $\begin{array}{c}\text { patchy } \\
\text { perivacuolar }\end{array}$ & $\begin{array}{c}\text { patchy } \\
\text { perivacuolar }\end{array}$ & extensive \\
\hline 6 & $\mathrm{~m}$ & 70 & 18 & $\mathrm{MV} 2 \mathrm{~K}+\mathrm{C}$ & yes, isolated & $\begin{array}{c}\text { patchy } \\
\text { perivacuolar }\end{array}$ & $\begin{array}{c}\text { patchy } \\
\text { perivacuolar }\end{array}$ & moderate \\
\hline $7^{*}$ & $\mathrm{~m}$ & 63 & 31 & $\mathrm{MV} 2 \mathrm{~K}+\mathrm{C}$ & yes & $\begin{array}{c}\text { patchy } \\
\text { perivacuolar }\end{array}$ & $\begin{array}{c}\text { patchy } \\
\text { perivacuolar }\end{array}$ & extensive \\
\hline 8 & $\mathrm{~m}$ & 57 & 2 & $\mathrm{MV} 2 \mathrm{~K}+\mathrm{C}$ & no & $\begin{array}{c}\text { deep } \\
\text { perineuronal }\end{array}$ & $\begin{array}{l}\text { patchy } \\
\text { perivacuolar }+ \\
\text { synaptic }\end{array}$ & $\begin{array}{c}\text { moderate } \\
\text { frontal, } \\
\text { extensive } \\
\text { occipital }\end{array}$ \\
\hline 9 & $\mathrm{f}$ & 73 & 9 & $\mathrm{MV} 2 \mathrm{~K}+\mathrm{C}$ & yes, few & $\begin{array}{c}\text { moderate deep } \\
\text { laminar }+ \\
\text { extensive patchy } \\
\text { perivacuolar }\end{array}$ & $\begin{array}{l}\text { moderate deep } \\
\text { laminar }+ \\
\text { extensive patchy } \\
\text { perivacuolar }\end{array}$ & $\begin{array}{l}\text { moderate- } \\
\text { extensive }\end{array}$ \\
\hline 10 & $\mathrm{~m}$ & 57 & 57 & $\mathrm{MV} 2 \mathrm{~K}+\mathrm{C}$ & yes, isolated & $\begin{array}{c}\text { moderate deep } \\
\text { laminar + focal } \\
\text { patchy } \\
\text { perivacuolar }\end{array}$ & $\begin{array}{l}\text { moderate deep } \\
\text { laminar }+ \\
\text { extensive patchy } \\
\text { perivacuolar }\end{array}$ & $\begin{array}{l}\text { moderate } \\
\text { frontal, } \\
\text { extensive } \\
\text { occipital }\end{array}$ \\
\hline 1 & $\mathrm{f}$ & 79 & 5 & $\mathrm{MM} 2 \mathrm{C}+1$ & yes & $\begin{array}{c}\text { patchy } \\
\text { perivacuolar }\end{array}$ & $\begin{array}{c}\text { patchy } \\
\text { perivacuolar }\end{array}$ & extensive \\
\hline 2 & $\mathrm{f}$ & 52 & 58 & MM2C & yes, extensive & $\begin{array}{c}\text { patchy } \\
\text { perivacuolar }\end{array}$ & $\begin{array}{c}\text { patchy } \\
\text { perivacuolar }\end{array}$ & extensive \\
\hline 3 & $\mathrm{f}$ & 80 & 2 & MM2C & yes, few & $\begin{array}{c}\text { patchy } \\
\text { perivacuolar }\end{array}$ & $\begin{array}{c}\text { patchy } \\
\text { perivacuolar }\end{array}$ & $\begin{array}{c}\text { moderate } \\
\text { frontal, } \\
\text { extensive } \\
\text { occipital } \\
\end{array}$ \\
\hline 4 & $\mathrm{~m}$ & 78 & 22 & MMC & yes & $\begin{array}{c}\text { patchy } \\
\text { perivacuolar }\end{array}$ & $\begin{array}{c}\text { patchy } \\
\text { perivacuolar }\end{array}$ & extensive \\
\hline 5 & $\mathrm{f}$ & 60 & 11 & MM2C + 1 & yes & $\begin{array}{c}\text { patchy } \\
\text { perivacuolar }\end{array}$ & $\begin{array}{l}\text { patchy } \\
\text { perivacuolar }+ \\
\text { synaptic }\end{array}$ & extensive \\
\hline 6 & $\mathrm{f}$ & 76 & 12 & MM2C & yes, few & $\begin{array}{c}\text { patchy } \\
\text { perivacuolar }\end{array}$ & $\begin{array}{c}\text { patchy } \\
\text { perivacuolar }\end{array}$ & moderate \\
\hline 7 & $\mathrm{f}$ & 77 & 3 & MM2C & no & $\begin{array}{c}\text { patchy } \\
\text { perivacuolar }\end{array}$ & $\begin{array}{c}\text { patchy } \\
\text { perivacuolar }\end{array}$ & $\begin{array}{l}\text { moderate- } \\
\text { extensive }\end{array}$ \\
\hline 8 & $\mathrm{f}$ & 64 & 12 & MM2C & yes & $\begin{array}{c}\text { patchy } \\
\text { perivacuolar }\end{array}$ & $\begin{array}{c}\text { patchy } \\
\text { perivacuolar }\end{array}$ & $\begin{array}{c}\text { moderate } \\
\text { frontal, } \\
\text { extensive } \\
\text { occipital } \\
\end{array}$ \\
\hline 9 & $\mathrm{f}$ & 60 & 2 & $\mathrm{MM} 2 \mathrm{C}+1$ & yes & $\begin{array}{c}\text { patchy } \\
\text { perivacuolar }\end{array}$ & $\begin{array}{l}\text { patchy } \\
\text { perivacuolar }+ \\
\text { synaptic }\end{array}$ & $\begin{array}{c}\text { extensive } \\
\text { frontal, } \\
\text { moderate } \\
\text { occipital }\end{array}$ \\
\hline 10 & $\mathrm{f}$ & 51 & 11 & $\mathrm{MM} 2 \mathrm{C}$ & yes & $\begin{array}{c}\text { patchy } \\
\text { perivacuolar }\end{array}$ & $\begin{array}{c}\text { patchy } \\
\text { perivacuolar }\end{array}$ & extensive \\
\hline
\end{tabular}




\section{Results}

We identified $\operatorname{PrP}^{\mathrm{d}}$ pathology in white matter glial cells with oligodendroglia morphology only in cases with the MM2C (+1) $(90 \%$ MM2C, $27 \%$ MM1 + 2C) and the mixed MV2K $+\mathrm{C}$ histotype (70\%). The oligodendroglial pathology was particularly evident in cases with extensive patchy-perivacuolar PrP deposits in the overlying cortex (Figure $1 \mathrm{H}, \mathrm{I})$. The deposits were primarily cytoplasmatic and ring- or comma-shaped (Figure 1J-L), and were associated with fine-punctuate $\mathrm{PrP}^{\mathrm{d}}$ deposits in the white matter reminiscent of axonal deposits. Moreover, oligodendroglial $\mathrm{PrP}^{\mathrm{d}}$ deposits were comparable with coiled bodies observed in the four-repeat tauopathies, such as progressive supranuclear palsy, corticobasal degeneration or argyrophilic grain disease, or even glial cytoplasmic alphasynuclein inclusions of MSA, as some $\mathrm{PrP}^{\mathrm{d}}$ aggregates appeared coarser or microglobular. We did not observe an obvious nuclear $\operatorname{PrP}^{\mathrm{d}}$ immunoreactivity. In contrast to tau and alphasynuclein inclusions in oligodendroglia, white matter oligodendroglial $\operatorname{PrP}^{\mathrm{d}}$ pathology was not visible in immunostaining for p62/ubiquitin and these deposits were not argyrophilic.

A comparable immunoreactivity pattern was identified when applying different antiPrP antibodies directed to different epitopes such as KG9, 6H4 and L42, while they were not well identified with the 3F4 antibody, which had a poorer performance globally. In mixed MM/MV1+2C cases, only when a high amount of patchy-perivacuolar deposits were present did single oligodendrocytes harbor ring-shaped and granular cytoplasmic $\mathrm{PrP}^{\mathrm{d}}$ immunoreactivity. In contrast, cases with only focal confluent vacuoles and focal patchy $\operatorname{PrP}^{\mathrm{d}}$ deposits had no obvious oligodendroglial PrP inclusions (Table 1).

Oligodendroglial $\mathrm{PrP}^{\mathrm{d}}$ accumulation was observed only in the subcortical white matter along axonal profiles, but was not visible in perivascular oligodendrocytes or perineuronal satellite oligodendrocytes within the cortex. Double immunofluorescence combining anti-TPPP/p25 and PrP (12F10) antibodies supported the oligodendroglial nature of cells harboring $\mathrm{PrP}^{\mathrm{d}}$ aggregates (Figure $1 \mathrm{M}, \mathrm{N}$ ). In contrast, GFAP+ astrocytes did not show $\mathrm{PrP}^{\mathrm{d}}$ accumulation within their cytoplasm (Figure 1O). The presence of oligodendroglial pathology was not related to age or sex, but was more frequently observed in cases with a longer disease duration. We could not identify oligodendroglial inclusions in MM/MV1, VV2 or pure MV2K cases. In MM/MV1 cases with extensive diffuse synaptic PrP deposits and prominent spongiform change, neuronal loss and gliosis, some ramified microglial cells at the cortico-subcortical boundary contained granular cytoplasmic PrP, but were not seen beyond that boundary. 

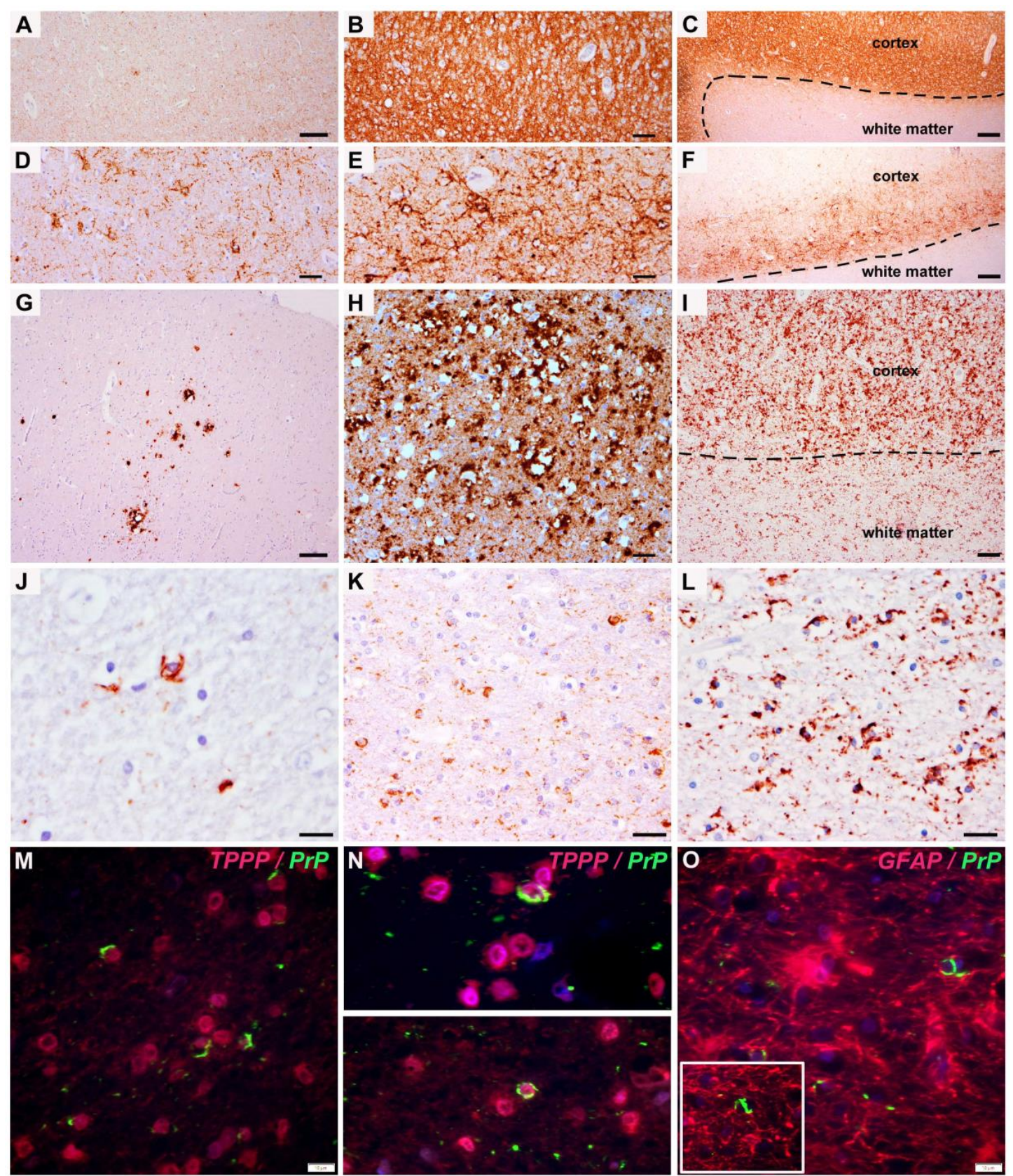

Figure 1. PrPd immunoreactivity patterns. A-C (MM1/MV1): Diffuse synaptic pattern, characteristic of MM/MV1 histotype ranging from mild (A) to extensive (B) deposits. (C) A relatively distinct border between cortical grey matter (upper part) and white matter (lower part) is observed in this subtype. No oligodendroglial pathology is usually detected. (D-F) (VV2/MV2): Perineuronal pattern, characteristic of VV2 (and MV2K) histotype in its milder (D) and more intense (E) form. This pattern has a typical deep laminar distribution (F). Here also, the border between cortical grey and white matter is relatively sharp, although some neuronal processes extend into the immediately underlying white matter. G-I (MM2C): Patchy perivacuolar pattern, characteristic of MM2C histotype, can appear as a focal feature (G), usually in mixed MM1+2C forms) or be widespread in bona fide MM2C $(\mathbf{H})$. In some cases with very extensive cortical pathology, there might be a blurring of the grey/white matter boundary (I) and pathological PrP deposits can be abundant in the underlying white matter. (J-L) At higher magnification, PrP deposits can be identified in single (J) or multiple (K,L) white matter glial cells with oligodendroglial morphology. These aggregates show ring-like, coiled-body-like or more amorphous morphologies. (M-O) Double immunofluorescence: semilunar or ring-like glial $\mathrm{PrP}^{\mathrm{d}}$ deposits (green signal) were clearly within TPPP/p25 positive oligodendrocyte (M, N: red signal; Patient 7/MV2K+2C from Table 1) but not within GFAP positive astrocytes (O, red signal); true co-localization of $\mathrm{PrP}^{\mathrm{d}}$, however, occurred rarely with TPPP/p25 (lower panel in N). Scale bars: $A$, G: $50 \mu \mathrm{m} ; \mathrm{B}, \mathrm{D}, \mathrm{E}, \mathrm{H}: 20 \mu \mathrm{m} ; \mathrm{C}, \mathrm{F}, \mathrm{I}: 100 \mu \mathrm{m} ; \mathrm{J}: 15 \mu \mathrm{m} ; \mathrm{K}, \mathrm{L}, \mathrm{M}, \mathrm{N}, \mathrm{O}: 10 \mu \mathrm{m}$. 


\section{Discussion}

Our findings demonstrate that particular CJD histotypes, mainly those dominated by $\operatorname{PrP}^{\mathrm{d}}$ type 2 with abundant patchy-perivacuolar deposits in the cortex (M2C "strain"), may be prone to accumulate $\mathrm{PrP}^{\mathrm{d}}$ in oligodendroglial cells, particularly in patients with a long disease duration, and support earlier evidence of oligodendroglial involvement in some CJD cases. The presence of oligodendroglial $\mathrm{PrP}^{\mathrm{d}}$ was not related to the cortical area analysed (frontal or occipital) but to the $\mathrm{PrP}^{\mathrm{d}}$ deposition pattern and its intensity in the overlying cortex.

MM2C patients typically manifest disease at older ages, present with progressive dementia, and have longer disease durations than classical MM1 or VV2 patients [7,8]. The total amounts of 14-3-3 and tau proteins are usually increased in CSF, but the RT-QuIC assay for PrP may result negative [9]. Patients are therefore frequently misdiagnosed with Alzheimer's disease or vascular/mixed dementia [8]. Neuropathological studies reveal prominent cortical pathology with large confluent vacuoles and patchy-perivacuolar $\mathrm{PrP}^{\mathrm{d}}$ deposits with a relative sparing of the brainstem and cerebellum. Cortical MRI hyperintensities correlate well with this anatomical distribution. About one third of MM/MV1 patients also show focal areas of confluent vacuoles with a patchy-perivacuolar PrP deposition pattern [10], but are clinically indistinguishable from more "pure" MM1 cases, except maybe for the presence of more pronounced cortical hyperintensities in MRI in the areas with mixed pathology. MM2C features can also accompany some MV2K cases and here again, cortical hyperintensities may correlate to the foci of large confluent vacuoles [11,12].

By $\operatorname{PrP}^{\mathrm{d}}$ immunohistochemistry, the cortical-subcortical boundary appears relatively sharply demarcated in typical MM/MV1 (Figure 1C), while in extensive MM2C patterns it appears blurred and $\mathrm{PrP}^{\mathrm{d}}$ deposits frequently extend into the white matter (Figure 1I). The deep laminar perineuronal pattern in VV2 may also project the delicate neuronal processes into the underlying white matter (Figure 1F) or show plaque-like or coarse deposits along the axons, but $\operatorname{PrP}^{\mathrm{d}}$ does not accumulate in oligodendroglial cytoplasm. At the cortico-subcortical boundary of some MM1/MV1 cases with extensive PrPd pathology, microglia may harbor PrP deposits, as they do in the cortex [3,13]. However, in more distant white matter, we observed non-argyrophilic and p62/ubiquitin negative, morphologically "coil-like" bodies in oligodendrocytes only in M2C cases.

Glial cells play an important role in neuronal homeostasis, connectivity and plasticity [14-16]. Oligodendrocytes, besides insulating and supporting axons through the myelin sheath are also important regulators of signal transmission and synaptic function. Oligodendrocytes also interact with GABAergic interneurons of the cortex, which represent almost $50 \%$ of myelin content in the upper cortical layers [17-19], and are frequently affected in the early disease stages of CJD [20-23]. As both oligodendrocytes and axons need their mutual input for proper functioning, it may be that the toxic properties of aggregated $\operatorname{PrP}^{\mathrm{d}}$ or the loss of $\operatorname{PrP}^{\mathrm{c}}$ function alter the axon-oligodendrocyte interaction. We observed $\mathrm{PrP}^{\mathrm{d}}$ aggregates in oligodendrocytes in white matter, but not in cortical satellite oligodendrocytes. White matter myelin is traditionally considered to ensheath the axons of pyramidal neurons. Therefore, $\mathrm{PrP}^{\mathrm{d}}$ aggregates transported along the axon might be taken-up by oligodendrocytes, a mechanism that has been suggested for oligodendrocyte alpha-synuclein accumulation in multiple system atrophy [24]. The axonal transport of $\mathrm{PrP}^{\mathrm{d}}$ is also known to occur in the VV2 subtype where $\mathrm{PrP}^{\mathrm{d}}$ accumulation can be identified along white matter axons and in perivascular areas $[7,25,26]$. Whether the MM2C patchy perivacuolar pattern affects a particular neuronal subtype that interacts more closely with oligodendrocytes, or whether in the M2C strain-specific $\mathrm{PrP}^{\mathrm{d}}$ molecules transported from these cortical deposits in axons are more prone to be taken up by oligodendroglia in the white matter, is not clear and deserves further investigation.

In the late 1990s, El Hachimi et al. [27] identified $\mathrm{PrP}^{\mathrm{d}}$ deposits in the white matter along myelin sheaths and in oligodendrocytes in four CJD cases (unspecified subtypes). Ultrastructural studies revealed the presence of dense osmiophilic, amorphous, partly 
fibrillar material associated with the lysosomes of oligodendrocytes. Moreover, Andres Benito et al. [28] reported an altered gene expression profile specific to astrocytes, oligodendrocytes and myelin in the frontal cortex of SCJD (7 MM1, 10 VV2), supporting the notion that molecular deficits linked to energy metabolism and solute transport in astrocytes and oligodendrocytes, in addition to neurons, may be relevant in the pathogenesis of cortical lesions in CJD. The authors also made similar observations in a murine CJD model [29].

In animals, a ramified astroglial PrP pattern is described for BSE and scrapie [20-32]. Particularly, in experimental TSE, the H-type BSE cases have been reported to show widespread glial labelling throughout the white matter of the spinal cord and the cerebellum [33]. Whether the similarities with some particular human disease forms with rare phenotypes might indicate some environmental influence on the disease phenotype remains unclear.

\section{Conclusions}

Oligodendroglial $\operatorname{PrP}^{\mathrm{d}}$ pathology may be detected in the white matter of SCJD, particularly in those subtypes with abundant patchy-perivacuolar PrP type 2 aggregates (M2C "strain"), which are usually, but not necessarily, associated with longer disease duration.

Author Contributions: Conceptualization, E.G. and G.G.K.; methodology, E.G., S.K., G.R. (Gerda Ricken); formal analysis and investigation, E.G., S.K., N.V.-R., G.R. (Gerda Ricken), G.R. (Günther Regelsberger), T.S., O.K., M.L., H.B., G.G.K.; resources, E.G., O.K., M.L., H.B., G.G.K.; writingoriginal draft preparation, E.G.; writing—review and editing, E.G., S.K., N.V.-R., G.R. (Gerda Ricken); G.R. (Günther Regelsberger), T.S., O.K., M.L., H.B., G.G.K.; supervision, H.B., G.G.K.; project administration, E.G.; funding acquisition, E.G. All authors have read and agreed to the published version of the manuscript.

Funding: The Austrian Reference Center for Human Prion diseases (OERPE) is funded by the Austrian Federal Ministry of Social Affairs, Health, Care and Consumer Protection. E.G. holds a grant from the "Medizinisch-Wissenschaftlichen Fonds des Burgermeisters der Stadt Wien" (project no. 18097) which supported part of the studies. S.K. holds a grant from the "Hochschuljubiläumsfonds der Stadt Wien" (project no. H-283459/2019).

Institutional Review Board Statement: The study was conducted according to the guidelines of the Declaration of Helsinki and approved by the Ethics Committee of the Medical University of Vienna (EK1454/2018, EK1636/2019).

Informed Consent Statement: Data were gathered in the frame of the Austrian Human Prion Disease Surveillance Program. In Austria, autopsy is mandatory for disease confirmation. Therefore, and as this was an anonymized postmortem study, there is no specific written informed consent from patients.

Data Availability Statement: The data presented in this study are available on request from the corresponding author. Some patients' data are not publicly available due to privacy reasons.

Acknowledgments: We thank the patients and their families for their support in CJD research. We thank the laboratory technicians from the Division of Neuropathology and Neurochemistry, Medical University of Vienna, for excellent technical and laboratory support. We also thank Daniel C. Bradley for English editing.

Conflicts of Interest: The authors declare no conflict of interest. The funders had no role in the design of the study; in the collection, analyses, or interpretation of data; or in the writing of the manuscript.

\section{References}

1. Prusiner, S.B. Molecular biology of prion diseases. Science 1991, 252, 1515-1522. [CrossRef]

2. Ironside, J.W.; Sutherland, K.; Bell, J.E.; McCardle, L.; Barrie, C.; Estebeiro, K.; Zeidler, M.; Will, R.G. A new variant of CreutzfeldtJakob disease: Neuropathological and clinical features. In Cold Spring Harbor Symposia on Quantitative Biology; Cold Spring Harbor Laboratory Press: New York, NY, USA, 1996; Volume 61, pp. 523-530.

3. Kovács, G.G.; Preusser, M.; Strohschneider, M.; Budka, H. Subcellular localization of disease-associated prion protein in the human brain. Am. J. Pathol. 2005, 166, 287-294. [CrossRef] 
4. Bošnjak, M.; Zupan, A.; Fiorini, M.; Popović, K.Š.; Popović, M. A case of MV2K subtype of sporadic Creutzfeldt-Jakob disease with florid-like plaques: Similarities and differences to variant Creutzfeldt-Jakob disease. Neuropathology 2020, 40, 389-398. [CrossRef]

5. Fernández-Vega, I.; Díaz-Lucena, D.; Azkune Calle, I.; Geijo, M.; Juste, R.A.; Llorens, F.; Vicente Etxenausia, I.; Santos-Juanes, J.; Zarranz Imirizaldu, J.J.; Ferrer, I. Sporadic Creutzfeldt-Jakob disease with glial $\mathrm{PrP}^{\text {Res }}$ nuclear and perinuclear immunoreactivity. Neuropathology 2018, 38, 561-567. [CrossRef]

6. Lehotzky, A.; Lau, P.; Tokési, N.; Muja, N.; Hudson, L.D.; Ovádi, J. Tubulin polymerization-promoting protein (TPPP/p25) is critical for oligodendrocyte differentiation. Glia 2010, 58, 157-168. [CrossRef]

7. Parchi, P.; Giese, A.; Capellari, S.; Brown, P.; Schulz-Schaeffer, W.; Windl, O.; Zerr, I.; Budka, H.; Kopp, N.; Piccardo, P.; et al. Classification of sporadic Creutzfeldt-Jakob disease based on molecular and phenotypic analysis of 300 subjects. Ann. Neurol. 1999, 46, 224-233. [CrossRef]

8. Krasnianski, A.; Meissner, B.; Schulz-Schaeffer, W.; Kallenberg, K.; Bartl, M.; Heinemann, U.; Varges, D.; Kretzschmar, H.A.; Zerr, I. Clinical features and diagnosis of the MM2 cortical subtype of sporadic Creutzfeldt-Jakob disease. Arch. Neurol. 2006, 63, 876-880. [CrossRef]

9. Abu-Rumeileh, S.; Baiardi, S.; Polischi, B.; Mammana, A.; Franceschini, A.; Green, A.; Capellari, S.; Parchi, P. Diagnostic value of surrogate CSF biomarkers for Creutzfeldt-Jakob disease in the era of RT-QuIC. J. Neurol. 2019, 266, 3136-3143. [CrossRef] [PubMed]

10. Parchi, P.; Strammiello, R.; Notari, S.; Giese, A.; Langeveld, J.P.; Ladogana, A.; Zerr, I.; Roncaroli, F.; Cras, P.; Ghetti, B.; et al. Incidence and spectrum of sporadic Creutzfeldt-Jakob disease variants with mixed phenotype and co-occurrence of PrPSc types: An updated classification. Acta Neuropathol. 2009, 118, 659-671. [CrossRef]

11. Grau-Rivera, O.; Sánchez-Valle, R.; Bargalló, N.; Lladó, A.; Gaig, C.; Nos, C.; Ferrer, I.; Graus, F.; Gelpi, E. Sporadic MM2thalamic + cortical Creutzfeldt-Jakob disease: Utility of diffusion tensor imaging in the detection of cortical involvement In Vivo. Neuropathology 2016, 36, 199-204. [CrossRef] [PubMed]

12. Iwasaki, Y.; Saito, Y.; Aiba, I.; Kobayashi, A.; Mimuro, M.; Kitamoto, T.; Yoshida, M. An autopsied case of MV2K+ C-type sporadic Creutzfeldt-Jakob disease presenting with widespread cerebral cortical involvement and Kuru plaques. Neuropathology 2017, 37, 241-248. [CrossRef] [PubMed]

13. Franceschini, A.; Strammiello, R.; Capellari, S.; Giese, A.; Parchi, P. Regional pattern of microgliosis in sporadic Creutzfeldt-Jakob disease in relation to phenotypic variants and disease progression. Neuropathol. Appl. Neurobiol. 2018, 44, 574-589. [CrossRef] [PubMed]

14. Lee, Y.; Morrison, B.M.; Li, Y.; Lengacher, S.; Farah, M.H.; Hoffman, P.N.; Liu, Y.; Tsingalia, A.; Jin, L.; Zhang, P.-W.; et al. Oligodendroglia metabolically support axons and contribute to neurodegeneration. Nat. Cell Biol. 2012, 487, 443-448. [CrossRef]

15. Jang, M.; Gould, E.; Xu, J.; Kim, E.J.; Kim, J.H. Oligodendrocytes regulate presynaptic properties and neurotransmission through BDNF signaling in the mouse brainstem. eLife 2019, 8, e42156. [CrossRef] [PubMed]

16. Fawcett, J.W.; Oohashi, T.; Pizzorusso, T. The roles of perineuronal nets and the perinodal extracellular matrix in neuronal function. Nat. Rev. Neurosci. 2019, 20, 451-465. [CrossRef]

17. Micheva, K.D.; Wolman, D.; Mensh, B.D.; Pax, E.; Buchanan, J.; Smith, S.J.; Bock, D.D. A large fraction of neocortical myelin ensheathes axons of local inhibitory neurons. eLife 2016, 5, e15784. [CrossRef]

18. Habermacher, C.; Angulo, M.C.; Benamer, N. Glutamate versus GABA in neuron-oligodendroglia communication. Glia 2019, 67, 2092-2106. [CrossRef]

19. Turko, P.; Groberman, K.; Browa, F.; Cobb, S.; Vida, I. Differential Dependence of GABAergic and Glutamatergic Neurons on Glia for the Establishment of Synaptic Transmission. Cereb. Cortex 2019, 29, 1230-1243. [CrossRef] [PubMed]

20. Ferrer, I.; Casas, R.; Rivera, R. Parvalbumin-immunoreactive cortical neurons in Creutzfeldt-Jakob disease. Ann. Neurol. 1993, 34, 864-866. [CrossRef] [PubMed]

21. Guentchev, M.; Groschup, M.H.; Kordek, R.; Liberski, P.P.; Budka, H. Severe, early and selective loss of a subpopulation of GABAergic inhibitory neurons in experimental transmissible spongiform encephalopathies. Brain Pathol. 1998, 8, 615-623. [CrossRef]

22. Belichenko, P.V.; Miklossy, J.; Belser, B.; Budka, H.; Celio, M.R. Early destruction of the extracellular matrix around parvalbuminimmunoreactive interneurons in Creutzfeldt-Jakob disease. Neurobiol. Dis. 1999, 6, 269-279. [CrossRef]

23. Guentchev, M.; Hainfellner, J.A.; Trabattoni, G.R.; Budka, H. Distribution of parvalbumin-immunoreactive neurons in brain correlates with hippocampal and temporal cortical pathology in Creutzfeldt-Jakob disease. J. Neuropathol. Exp. Neurol. 1997, 56, 1119-1124. [CrossRef] [PubMed]

24. Wenning, G.K.; Stefanova, N.; Jellinger, K.A.; Poewe, W.; Schlossmacher, M.G. Multiple system atrophy: A primary oligodendrogliopathy. Ann. Neurol. 2008, 64, 239-246. [CrossRef] [PubMed]

25. Parchi, P.; de Boni, L.; Saverioni, D.; Cohen, M.L.; Ferrer, I.; Gambetti, P.; Gelpi, E.; Giaccone, G.; Hauw, J.J.; Höftberger, R.; et al. Consensus classification of human prion disease histotypes allows reliable identification of molecular subtypes: An inter-rater study among surveillance centres in Europe and USA. Acta Neuropathol. 2012, 124, 517-529. [CrossRef] [PubMed]

26. Kovacs, G.G.; Head, M.W.; Bunn, T.; Laszlo, L.; Will, R.G.; Ironside, J.W. Clinicopathological phenotype of codon 129 valine homozygote sporadic Creutzfeldt-Jakob disease. Neuropathol. Appl. Neurobiol. 2000, 26, 463-472. [CrossRef] [PubMed] 
27. El Hachimi, K.H.; Chaunu, M.P.; Brown, P.; Foncin, J.F. Modifications of oligodendroglial cells in spongiform encephalopathies. Exp. Neurol. 1998, 154, 23-30. [CrossRef]

28. Andres Benito, P.; Dominguez Gonzalez, M.; Ferrer, I. Altered gene transcription linked to astrocytes and oligodendrocytes in frontal cortex in Creutzfeldt-Jakob disease. Prion 2018, 12, 216-225. [CrossRef]

29. Andrés-Benito, P.; Carmona, M.; Douet, J.Y.; Cassard, H.; Andreoletti, O.; Ferrer, I. Differential astrocyte and oligodendrocyte vulnerability in murine Creutzfeldt-Jakob disease. Prion 2021, 15, 112-120. [CrossRef]

30. Casalone, C.; Caramelli, M.; Crescio, M.I.; Spencer, Y.I.; Simmons, M.M. BSE immunohistochemical patterns in the brainstem: A comparison between UK and Italian cases. Acta Neuropathol. 2006, 111, 444-449. [CrossRef]

31. Simmons, M.M.; Spiropoulos, J.; Webb, P.R.; Spencer, Y.I.; Czub, S.; Mueller, R.; Davis, A.; Arnold, M.E.; Marsh, S.; Hawkins, S.A.; et al. Experimental classical bovine spongiform encephalopathy: Definition and progression of neural PrP immunolabeling in relation to diagnosis and disease controls. Vet. Pathol. 2011, 48, 948-963. [CrossRef]

32. Spiropoulos, J.; Casalone, C.; Caramelli, M.; Simmons, M.M. Immunohistochemistry for PrPSc in natural scrapie reveals patterns which are associated with the PrP genotype. Neuropathol. Appl. Neurobiol. 2007, 33, 398-409. [CrossRef] [PubMed]

33. Konold, T.; Bone, G.E.; Clifford, D.; Chaplin, M.J.; Cawthraw, S.; Stack, M.J.; Simmons, M.M. Experimental H-type and L-type bovine spongiform encephalopathy in cattle: Observation of two clinical syndromes and diagnostic challenges. BMC Vet. Res. 2012, 8, 22. [CrossRef] [PubMed] 